\title{
Measurement of $\sigma_{\Lambda^{0}} / \sigma_{\bar{B}^{0}} \times \mathcal{B}\left(\Lambda_{b}^{0} \rightarrow \Lambda_{c}^{+} \pi^{-}\right) / \mathcal{B}\left(\bar{B}^{0} \rightarrow D^{+} \pi^{-}\right)$ in $p \bar{p}$ Collisions at $\sqrt{s}=1.96 \mathrm{TeV}$
}

A. Abulencia, ${ }^{23}$ J. Adelman, ${ }^{13}$ T. Affolder, ${ }^{10}$ T. Akimoto, ${ }^{55}$ M. G. Albrow, ${ }^{16}$ D. Ambrose, ${ }^{16}$ S. Amerio, ${ }^{43}$ D. Amidei, ${ }^{34}$ A. Anastassov, ${ }^{52}$ K. Anikeev ${ }^{16}$ A. Annovi, ${ }^{18}$ J. Antos, ${ }^{1}$ M. Aoki, ${ }^{55}$ G. Apollinari, ${ }^{16}$ J.-F. Arguin, ${ }^{33}$ T. Arisawa, ${ }^{57}$ A. Artikov, ${ }^{14}$ W. Ashmanskas, ${ }^{16}$ A. Attal,${ }^{8}$ F. Azfar, ${ }^{42}$ P. Azzi-Bacchetta,${ }^{43}$ P. Azzurri, ${ }^{46}$ N. Bacchetta, ${ }^{43}$ W. Badgett, ${ }^{16}$ A. Barbaro-Galtieri, ${ }^{28}$ V. E. Barnes, ${ }^{48}$ B. A. Barnett, ${ }^{24}$ S. Baroiant, ${ }^{7}$ V. Bartsch, ${ }^{30}$ G. Bauer,${ }^{32}$ F. Bedeschi, ${ }^{46}$ S. Behari, ${ }^{24}$ S. Belforte ${ }^{54}$ G. Bellettini, ${ }^{46}$ J. Bellinger, ${ }^{59}$ A. Belloni, ${ }^{32}$ D. Benjamin, ${ }^{15}$ A. Beretvas, ${ }^{16}$ J. Beringer ${ }^{28}$ T. Berry, ${ }^{29}$ A. Bhatti, ${ }^{50}$ M. Binkley, ${ }^{16}$ D. Bisello, ${ }^{43}$ R. E. Blair, ${ }^{2}$ C. Blocker,${ }^{6}$ B. Blumenfeld,${ }^{24}$ A. Bocci, ${ }^{15}$ A. Bodek, ${ }^{49}$ V. Boisvert, ${ }^{49}$ G. Bolla, ${ }^{48}$ A. Bolshov, ${ }^{32}$ D. Bortoletto, ${ }^{48}$ J. Boudreau, ${ }^{47}$ A. Boveia, ${ }^{10}$ B. Brau, ${ }^{10}$ L. Brigliadori, ${ }^{5}$ C. Bromberg, ${ }^{35}$ E. Brubaker, ${ }^{13}$ J. Budagov, ${ }^{14}$ H. S. Budd,${ }^{49}$ S. Budd, ${ }^{23}$ S. Budroni, ${ }^{46}$ K. Burkett, ${ }^{16}$ G. Busetto, ${ }^{43}$ P. Bussey,${ }^{20}$ K. L. Byrum, ${ }^{2}$ S. Cabrera, ${ }^{15}$ M. Campanelli, ${ }^{19}$ M. Campbell, ${ }^{34}$ F. Canelli, ${ }^{16}$ A. Canepa, ${ }^{48}$ S. Carillo, ${ }^{17}$ D. Carlsmith, ${ }^{59}$ R. Carosi ${ }^{46}$ M. Casarsa,${ }^{54}$ A. Castro, ${ }^{5}$ P. Catastini,${ }^{46}$ D. Cauz,${ }^{54}$ M. Cavalli-Sforza, ${ }^{3}$ A. Cerri, ${ }^{28}$ L. Cerrito, ${ }^{42}$ S. H. Chang, ${ }^{27}$ Y. C. Chen, ${ }^{1}$ M. Chertok, ${ }^{7}$ G. Chiarelli, ${ }^{46}$ G. Chlachidze, ${ }^{14}$ F. Chlebana, ${ }^{16}$ I. Cho, ${ }^{27}$ K. Cho, ${ }^{27}$ D. Chokheli, ${ }^{14}$ J. P. Chou, ${ }^{21}$ G. Choudalakis, ${ }^{32}$ S. H. Chuang, ${ }^{59}$ K. Chung, ${ }^{12}$ W. H. Chung, ${ }^{59}$ Y. S. Chung, ${ }^{49}$ M. Ciljak, ${ }^{46}$ C. I. Ciobanu, ${ }^{23}$ M. A. Ciocci, ${ }^{46}$ A. Clark, ${ }^{19}$ D. Clark, ${ }^{6}$ M. Coca,${ }^{15}$ G. Compostella, ${ }^{43}$ M. E. Convery,${ }^{50}$ J. Conway, ${ }^{7}$ B. Cooper, ${ }^{35}$ K. Copic,,${ }^{34}$ M. Cordelli, ${ }^{18}$ G. Cortiana ${ }^{43}$ F. Crescioli, ${ }^{46}$ C. Cuenca Almenar, ${ }^{7}$ J. Cuevas, ${ }^{11}$ R. Culbertson, ${ }^{16}$ J. C. Cully, ${ }^{34}$ D. Cyr,${ }^{59}$ S. DaRonco, ${ }^{43}$ S. D'Auria ${ }^{20}$ T. Davies,${ }^{20}$ M. D'Onofrio, ${ }^{3}$ D. Dagenhart, ${ }^{6}$ P. de Barbaro, ${ }^{49}$ S. De Cecco, ${ }^{51}$ A. Deisher,${ }^{28}$

G. De Lentdecker, ${ }^{49}$ M. Dell'Orso, ${ }^{46}$ F. Delli Paoli, ${ }^{43}$ L. Demortier, ${ }^{50}$ J. Deng, ${ }^{15}$ M. Deninno, ${ }^{5}$ D. De Pedis,${ }^{51}$ P. F. Derwent, ${ }^{16}$ G. P. Di Giovanni, ${ }^{44}$ C. Dionisi,${ }^{51}$ B. Di Ruzza,${ }^{54}$ J. R. Dittmann, ${ }^{4}$ P. DiTuro, ${ }^{52}$ C. Dörr, ${ }^{25}$ S. Donati,${ }^{46}$ M. Donega, ${ }^{19}$ P. Dong, ${ }^{8}$ J. Donini, ${ }^{43}$ T. Dorigo, ${ }^{43}$ S. Dube, ${ }^{52}$ J. Efron, ${ }^{39}$ R. Erbacher, ${ }^{7}$ D. Errede,${ }^{23}$ S. Errede,${ }^{23}$ R. Eusebi, ${ }^{16}$ H. C. Fang, ${ }^{28}$ S. Farrington, ${ }^{29}$ I. Fedorko, ${ }^{46}$ W. T. Fedorko, ${ }^{13}$ R. G. Feild, ${ }^{60}$ M. Feindt, ${ }^{25}$ J. P. Fernandez, ${ }^{31}$ R. Field, ${ }^{17}$ G. Flanagan, ${ }^{48}$ A. Foland, ${ }^{21}$ S. Forrester, ${ }^{7}$ G. W. Foster, ${ }^{16}$ M. Franklin, ${ }^{21}$ J. C. Freeman, ${ }^{28}$ I. Furic, ${ }^{13}$ M. Gallinaro,${ }^{50}$

J. Galyardt, ${ }^{12}$ J. E. Garcia, ${ }^{46}$ F. Garberson, ${ }^{10}$ A. F. Garfinkel, ${ }^{48}$ C. Gay,${ }^{60}$ H. Gerberich,${ }^{23}$ D. Gerdes,${ }^{34}$ S. Giagu, ${ }^{51}$ P. Giannetti, ${ }^{46}$ A. Gibson, ${ }^{28}$ K. Gibson, ${ }^{47}$ J. L. Gimmell, ${ }^{49}$ C. Ginsburg, ${ }^{16}$ N. Giokaris, ${ }^{14}$ M. Giordani,${ }^{54}$ P. Giromini, ${ }^{18}$ M. Giunta, ${ }^{46}$ G. Giurgiu, ${ }^{12}$ V. Glagolev, ${ }^{14}$ D. Glenzinski, ${ }^{16}$ M. Gold,${ }^{37}$ N. Goldschmidt,${ }^{17}$ J. Goldstein,${ }^{42}$ G. Gomez, ${ }^{11}$ G. Gomez-Ceballos, ${ }^{11}$ M. Goncharov, ${ }^{53}$ O. González, ${ }^{31}$ I. Gorelov, ${ }^{37}$ A. T. Goshaw, ${ }^{15}$ K. Goulianos, ${ }^{50}$ A. Gresele, ${ }^{43}$ M. Griffiths ${ }^{29}$ S. Grinstein ${ }^{21}$ C. Grosso-Pilcher, ${ }^{13}$ R. C. Group,${ }^{17}$ U. Grundler, ${ }^{23}$ J. Guimaraes da Costa, ${ }^{21}$

Z. Gunay-Unalan, ${ }^{35}$ C. Haber ${ }^{28}$ K. Hahn, ${ }^{32}$ S. R. Hahn, ${ }^{16}$ E. Halkiadakis, ${ }^{52}$ A. Hamilton, ${ }^{33}$ B.-Y. Han ${ }^{49}$ J. Y. Han, ${ }^{49}$ R. Handler, ${ }^{59}$ F. Happacher, ${ }^{18}$ K. Hara, ${ }^{55}$ M. Hare, ${ }^{56}$ S. Harper ${ }^{42}$ R. F. Harr, ${ }^{58}$ R. M. Harris,${ }^{16}$ M. Hartz, ${ }^{47}$

K. Hatakeyama ${ }^{50}$ J. Hauser, ${ }^{8}$ A. Heijboer, ${ }^{45}$ B. Heinemann, ${ }^{29}$ J. Heinrich,${ }^{45}$ C. Henderson, ${ }^{32}$ M. Herndon,${ }^{59}$ J. Heuser, ${ }^{25}$ D. Hidas, ${ }^{15}$ C. S. Hill, ${ }^{10}$ D. Hirschbuehl,${ }^{25}$ A. Hocker, ${ }^{16}$ A. Holloway, ${ }^{21}$ S. Hou, ${ }^{1}$ M. Houlden,${ }^{29}$ S.-C. Hsu, ${ }^{9}$

B. T. Huffman, ${ }^{42}$ R. E. Hughes,${ }^{39}$ U. Husemann, ${ }^{60}$ J. Huston, ${ }^{35}$ J. Incandela,${ }^{10}$ G. Introzzi,${ }^{46}$ M. Iori, ${ }^{51}$ Y. Ishizawa, ${ }^{55}$ A. Ivanov, ${ }^{7}$ B. Iyutin, ${ }^{32}$ E. James,${ }^{16}$ D. Jang,${ }^{52}$ B. Jayatilaka, ${ }^{34}$ D. Jeans,${ }^{51}$ H. Jensen, ${ }^{16}$ E. J. Jeon, ${ }^{27}$ S. Jindariani, ${ }^{17}$ M. Jones, ${ }^{48}$ K. K. Joo, ${ }^{27}$ S. Y. Jun, ${ }^{12}$ J. E. Jung, ${ }^{27}$ T. R. Junk ${ }^{23}$ T. Kamon, ${ }^{53}$ P. E. Karchin, ${ }^{58}$ Y. Kato, ${ }^{41}$ Y. Kemp, ${ }^{25}$ R. Kephart, ${ }^{16}$ U. Kerzel, ${ }^{25}$ V. Khotilovich, ${ }^{53}$ B. Kilminster, ${ }^{39}$ D. H. Kim ${ }^{27}$ H. S. Kim, ${ }^{27}$ J.E. Kim, ${ }^{27}$ M. J. Kim, ${ }^{12}$ S. B. Kim, ${ }^{27}$ S. H. Kim, ${ }^{55}$ Y. K. Kim,${ }^{13}$ N. Kimura, ${ }^{55}$ L. Kirsch, ${ }^{6}$ S. Klimenko, ${ }^{17}$ M. Klute, ${ }^{32}$ B. Knuteson, ${ }^{32}$ B. R. Ko, ${ }^{15}$ K. Kondo,${ }^{57}$ D. J. Kong, ${ }^{27}$ J. Konigsberg, ${ }^{17}$ A. Korytov, ${ }^{17}$ A. V. Kotwal, ${ }^{15}$ A. Kovalev, ${ }^{45}$ A. C. Kraan, ${ }^{45}$ J. Kraus, ${ }^{23}$ I. Kravchenko, ${ }^{32}$ M. Kreps, ${ }^{25}$ J. Kroll, ${ }^{45}$ N. Krumnack, ${ }^{4}$ M. Kruse, ${ }^{15}$ V. Krutelyov, ${ }^{10}$ T. Kubo, ${ }^{55}$ S. E. Kuhlmann, ${ }^{2}$ T. Kuhr, ${ }^{25}$ Y. Kusakabe, ${ }^{57}$ S. Kwang, ${ }^{13}$ A. T. Laasanen, ${ }^{48}$ S. Lai, ${ }^{33}$ S. Lami, ${ }^{46}$ S. Lammel, ${ }^{16}$ M. Lancaster, ${ }^{30}$ R. L. Lander, ${ }^{7}$ K. Lannon, ${ }^{39}$ A. Lath,${ }^{52}$ G. Latino, ${ }^{46}$ I. Lazzizzera, ${ }^{43}$ Y. Le,${ }^{24}$ T. LeCompte, ${ }^{2}$ J. Lee, ${ }^{49}$ J. Lee, ${ }^{27}$ Y. J. Lee,${ }^{27}$ S. W. Lee, ${ }^{53}$ R. Lefèvre, ${ }^{3}$ N. Leonardo ${ }^{32}$ S. Leone, ${ }^{46}$ S. Levy, ${ }^{13}$ J. D. Lewis, ${ }^{16}$ C. Lin, ${ }^{60}$ C. S. Lin, ${ }^{16}$ M. Lindgren, ${ }^{16}$ E. Lipeles, ${ }^{9}$ A. Lister, ${ }^{7}$ D. O. Litvintsev, ${ }^{16}$ T. Liu,${ }^{16}$ N. S. Lockyer, ${ }^{45}$ A. Loginov, ${ }^{36}$ M. Loreti ${ }^{43}$ P. Loverre, ${ }^{51}$ R.-S. Lu, ${ }^{1}$ D. Lucchesi, ${ }^{43}$ P. Lujan, ${ }^{28}$ P. Lukens, ${ }^{16}$ G. Lungu,${ }^{17}$ L. Lyons,${ }^{42}$ J. Lys,${ }^{28}$ R. Lysak,,${ }^{1}$ E. Lytken ${ }^{48}$ P. Mack,${ }^{25}$ D. MacQueen, ${ }^{33}$ R. Madrak ${ }^{16}$ K. Maeshima, ${ }^{16}$ K. Makhoul, ${ }^{32}$ T. Maki, ${ }^{22}$ P. Maksimovic, ${ }^{24}$ S. Malde, ${ }^{42}$ G. Manca, ${ }^{29}$ F. Margaroli, ${ }^{5}$ R. Marginean, ${ }^{16}$ C. Marino, ${ }^{25}$ C.P. Marino, ${ }^{23}$ A. Martin, ${ }^{60}$ M. Martin,${ }^{24}$ V. Martin, ${ }^{20}$ M. Martínez, ${ }^{3}$ T. Maruyama,${ }^{55}$ P. Mastrandrea, ${ }^{51}$

T. Masubuchi, ${ }^{55}$ H. Matsunaga,${ }^{55}$ M.E. Mattson,${ }^{58}$ R. Mazini, ${ }^{33}$ P. Mazzanti, ${ }^{5}$ K. S. McFarland,${ }^{49}$ P. McIntyre, ${ }^{53}$ R. McNulty, ${ }^{29}$ A. Mehta,${ }^{29}$ P. Mehtala, ${ }^{22}$ S. Menzemer, ${ }^{11}$ A. Menzione,${ }^{46}$ P. Merkel, ${ }^{48}$ C. Mesropian, ${ }^{50}$ A. Messina, ${ }^{35}$ T. Miao, ${ }^{16}$ N. Miladinovic, ${ }^{6}$ J. Miles,${ }^{32}$ R. Miller,${ }^{35}$ C. Mills, ${ }^{10}$ M. Milnik, ${ }^{25}$ A. Mitra, ${ }^{1}$ G. Mitselmakher, ${ }^{17}$ A. Miyamoto, ${ }^{26}$ S. Moed, ${ }^{19}$ N. Moggi,${ }^{5}$ B. Mohr,${ }^{8}$ R. Moore,${ }^{16}$ M. Morello, ${ }^{46}$ P. Movilla Fernandez ${ }^{28}$ J. Mülmenstädt, ${ }^{28}$ A. Mukherjee, ${ }^{16}$ 
Th. Muller, ${ }^{25}$ R. Mumford,${ }^{24}$ P. Murat,${ }^{16}$ J. Nachtman, ${ }^{16}$ A. Nagano, ${ }^{55}$ J. Naganoma, ${ }^{57}$ I. Nakano, ${ }^{40}$ A. Napier,${ }^{56}$ V. Necula, ${ }^{17}$ C. Neu, ${ }^{45}$ M. S. Neubauer, ${ }^{9}$ J. Nielsen, ${ }^{28}$ T. Nigmanov,${ }^{47}$ L. Nodulman, ${ }^{2}$ O. Norniella, ${ }^{3}$ E. Nurse,${ }^{30}$ S. H. Oh, ${ }^{15}$ Y.D. Oh,${ }^{27}$ I. Oksuzian, ${ }^{17}$ T. Okusawa, ${ }^{41}$ R. Oldeman, ${ }^{29}$ R. Orava, ${ }^{22}$ K. Osterberg, ${ }^{22}$ C. Pagliarone, ${ }^{46}$ E. Palencia, ${ }^{11}$ V. Papadimitriou, ${ }^{16}$ A. A. Paramonov, ${ }^{13}$ B. Parks, ${ }^{39}$ S. Pashapour, ${ }^{33}$ J. Patrick, ${ }^{16}$ G. Pauletta ${ }^{54}$ M. Paulini, ${ }^{12}$ C. Paus, ${ }^{32}$ D. E. Pellett, ${ }^{7}$ A. Penzo,${ }^{54}$ T. J. Phillips,${ }^{15}$ G. Piacentino, ${ }^{46}$ J. Piedra, ${ }^{44}$ L. Pinera,,${ }^{17}$ K. Pitts, ${ }^{23}$ C. Plager, ${ }^{8}$ L. Pondrom, ${ }^{59}$ X. Portell, ${ }^{3}$ O. Poukhov, ${ }^{14}$ N. Pounder,${ }^{42}$ F. Prakoshyn, ${ }^{14}$ A. Pronko, ${ }^{16}$ J. Proudfoot, ${ }^{2}$ F. Ptohos, ${ }^{18}$ G. Punzi, ${ }^{46}$ J. Pursley,${ }^{24}$ J. Rademacker, ${ }^{42}$ A. Rahaman, ${ }^{47}$ N. Ranjan, ${ }^{48}$ S. Rappoccio, ${ }^{21}$ B. Reisert,,${ }^{16}$ V. Rekovic, ${ }^{37}$ P. Renton, ${ }^{42}$ M. Rescigno, ${ }^{51}$ S. Richter, ${ }^{25}$ F. Rimondi, ${ }^{5}$ L. Ristori,${ }^{46}$ A. Robson, ${ }^{20}$ T. Rodrigo, ${ }^{11}$ E. Rogers, ${ }^{23}$ S. Rolli, ${ }^{56}$ R. Roser,,${ }^{16}$ M. Rossi, ${ }^{54}$ R. Rossin, ${ }^{17}$ A. Ruiz, ${ }^{11}$ J. Russ, ${ }^{12}$ V. Rusu, ${ }^{13}$ H. Saarikko, ${ }^{22}$ S. Sabik,${ }^{33}$ A. Safonov,${ }^{53}$ W. K. Sakumoto, ${ }^{49}$ G. Salamanna, ${ }^{51}$ O. Saltó, ${ }^{3}$ D. Saltzberg, ${ }^{8}$ C. Sánchez, ${ }^{3}$ L. Santi, ${ }^{54}$ S. Sarkar, ${ }^{51}$ L. Sartori, ${ }^{46}$ K. Sato, ${ }^{16}$ P. Savard, ${ }^{33}$ A. Savoy-Navarro, ${ }^{44}$ T. Scheidle, ${ }^{25}$ P. Schlabach, ${ }^{16}$ E. E. Schmidt, ${ }^{16}$ M. P. Schmidt,${ }^{60}$ M. Schmitt, ${ }^{38}$ T. Schwarz, ${ }^{7}$ L. Scodellaro, ${ }^{11}$ A. L. Scott, ${ }^{10}$ A. Scribano, ${ }^{46}$ F. Scuri, ${ }^{46}$ A. Sedov, ${ }^{48}$ S. Seidel,${ }^{37}$ Y. Seiya, ${ }^{41}$ A. Semenov,${ }^{14}$ L. Sexton-Kennedy, ${ }^{16}$ A. Sfyrla, ${ }^{19}$ M. D. Shapiro, ${ }^{28}$ T. Shears, ${ }^{29}$ P. F. Shepard, ${ }^{47}$ D. Sherman, ${ }^{21}$ M. Shimojima, ${ }^{55}$ M. Shochet, ${ }^{13}$ Y. Shon,${ }^{59}$ I. Shreyber, ${ }^{36}$

A. Sidoti, ${ }^{46}$ P. Sinervo, ${ }^{33}$ A. Sisakyan, ${ }^{14}$ J. Sjolin, ${ }^{42}$ A. J. Slaughter, ${ }^{16}$ J. Slaunwhite, ${ }^{39}$ K. Sliwa, ${ }^{56}$ J. R. Smith, ${ }^{7}$ F. D. Snider, ${ }^{16}$ R. Snihur, ${ }^{33}$ M. Soderberg, ${ }^{34}$ A. Soha,${ }^{7}$ S. Somalwar, ${ }^{52}$ V. Sorin, ${ }^{35}$ J. Spalding, ${ }^{16}$ F. Spinella, ${ }^{46}$ T. Spreitzer, ${ }^{33}$ P. Squillacioti ${ }^{46}$ M. Stanitzki, ${ }^{60}$ A. Staveris-Polykalas, ${ }^{46}$ R. St. Denis, ${ }^{20}$ B. Stelzer, ${ }^{8}$ O. Stelzer-Chilton, ${ }^{42}$ D. Stentz, ${ }^{38}$ J. Strologas,${ }^{37}$ D. Stuart, ${ }^{10}$ J. S. Suh,${ }^{27}$ A. Sukhanov, ${ }^{17}$ H. Sun,${ }^{56}$ T. Suzuki, ${ }^{55}$ A. Taffard, ${ }^{23}$ R. Takashima, ${ }^{40}$ Y. Takeuchi, ${ }^{55}$

K. Takikawa, ${ }^{55}$ M. Tanaka, ${ }^{2}$ R. Tanaka, ${ }^{40}$ M. Tecchio, ${ }^{34}$ P. K. Teng,,${ }^{1}$ K. Terashi,${ }^{50}$ J. Thom, ${ }^{16}$ A. S. Thompson, ${ }^{20}$ E. Thomson,${ }^{45}$ P. Tipton, ${ }^{60}$ V. Tiwari, ${ }^{12}$ S. Tkaczyk,${ }^{16}$ D. Toback,${ }^{53}$ S. Tokar, ${ }^{14}$ K. Tollefson, ${ }^{35}$ T. Tomura,${ }^{55}$ D. Tonelli, ${ }^{46}$ S. Torre, ${ }^{18}$ D. Torretta, ${ }^{16}$ S. Tourneur, ${ }^{44}$ W. Trischuk,${ }^{33}$ J. Tseng, ${ }^{32}$ R. Tsuchiya, ${ }^{57}$ S. Tsuno, ${ }^{40}$ N. Turini, ${ }^{46}$ F. Ukegawa ${ }^{55}$ T. Unverhau, ${ }^{20}$ S. Uozumi,${ }^{55}$ D. Usynin, ${ }^{45}$ S. Vallecorsa, ${ }^{19}$ N. van Remortel, ${ }^{22}$ A. Varganov, ${ }^{34}$ E. Vataga,${ }^{37}$ F. Vázquez, ${ }^{17}$ G. Velev, ${ }^{16}$ G. Veramendi, ${ }^{23}$ V. Veszpremi, ${ }^{48}$ R. Vidal, ${ }^{16}$ I. Vila, ${ }^{11}$ R. Vilar, ${ }^{11}$ T. Vine, ${ }^{30}$ I. Vollrath, ${ }^{33}$ I. Volobouev, ${ }^{28}$ G. Volpi ${ }^{46}$ F. Würthwein, ${ }^{9}$ P. Wagner, ${ }^{53}$ R. G. Wagner, ${ }^{2}$ R. L. Wagner, ${ }^{16}$ J. Wagner, ${ }^{25}$ W. Wagner, ${ }^{25}$ R. Wallny, ${ }^{8}$ S. M. Wang, ${ }^{1}$ A. Warburton, ${ }^{33}$ S. Waschke, ${ }^{20}$ D. Waters,${ }^{30}$ W. C. Wester III,${ }^{16}$ B. Whitehouse, ${ }^{56}$ D. Whiteson, ${ }^{45}$ A. B. Wicklund, ${ }^{2}$ E. Wicklund, ${ }^{16}$ G. Williams, ${ }^{33}$ H. H. Williams, ${ }^{45}$ P. Wilson, ${ }^{16}$ B. L. Winer, ${ }^{39}$ P. Wittich, ${ }^{16}$ S. Wolbers, ${ }^{16}$ C. Wolfe, ${ }^{13}$ T. Wright,${ }^{34}$ X. Wu, ${ }^{19}$ S. M. Wynne, ${ }^{29}$ A. Yagil,${ }^{16}$ K. Yamamoto, ${ }^{41}$ J. Yamaoka, ${ }^{52}$ T. Yamashita, ${ }^{40}$ C. Yang, ${ }^{60}$ U. K. Yang, ${ }^{13}$ Y. C. Yang, ${ }^{27}$ W. M. Yao, ${ }^{28}$ G. P. Yeh, ${ }^{16}$ J. Yoh, ${ }^{16}$ K. Yorita, ${ }^{13}$ T. Yoshida, ${ }^{41}$ G. B. Yu, ${ }^{49}$ I. Yu, ${ }^{27}$ S. S. Yu, ${ }^{16}$ J. C. Yun, ${ }^{16}$ L. Zanello, ${ }^{51}$ A. Zanetti, ${ }^{54}$ I. Zaw, ${ }^{21}$ X. Zhang, ${ }^{23}$ J. Zhou, ${ }^{52}$ and S. Zucchelli ${ }^{5}$

\title{
(CDF Collaboration)
}

\author{
${ }^{1}$ Institute of Physics, Academia Sinica, Taipei, Taiwan 11529, Republic of China \\ ${ }^{2}$ Argonne National Laboratory, Argonne, Illinois 60439, USA \\ ${ }^{3}$ Institut de Fisica d'Altes Energies, Universitat Autonoma de Barcelona, E-08193, Bellaterra (Barcelona), Spain \\ ${ }^{4}$ Baylor University, Waco, Texas 76798, USA \\ ${ }^{5}$ Istituto Nazionale di Fisica Nucleare, University of Bologna, I-40127 Bologna, Italy \\ ${ }^{6}$ Brandeis University, Waltham, Massachusetts 02254, USA \\ ${ }^{7}$ University of California, Davis, Davis, California 95616, USA \\ ${ }^{8}$ University of California, Los Angeles, Los Angeles, California 90024, USA \\ ${ }^{9}$ University of California, San Diego, La Jolla, California 92093, USA \\ ${ }^{10}$ University of California, Santa Barbara, Santa Barbara, California 93106, USA \\ ${ }^{11}$ Instituto de Fisica de Cantabria, CSIC-University of Cantabria, 39005 Santander, Spain \\ ${ }^{12}$ Carnegie Mellon University, Pittsburgh, Pennsylvania 15213, USA \\ ${ }^{13}$ Enrico Fermi Institute, University of Chicago, Chicago, Illinois 60637, USA \\ ${ }^{14}$ Joint Institute for Nuclear Research, RU-141980 Dubna, Russia \\ ${ }^{15}$ Duke University, Durham, North Carolina 27708, USA \\ ${ }^{16}$ Fermi National Accelerator Laboratory, Batavia, Illinois 60510, USA \\ ${ }^{17}$ University of Florida, Gainesville, Florida 32611, USA \\ ${ }^{18}$ Laboratori Nazionali di Frascati, Istituto Nazionale di Fisica Nucleare, I-00044 Frascati, Italy \\ ${ }^{19}$ University of Geneva, CH-1211 Geneva 4, Switzerland \\ ${ }^{20}$ Glasgow University, Glasgow G12 8QQ, United Kingdom \\ ${ }^{21}$ Harvard University, Cambridge, Massachusetts 02138, USA \\ ${ }^{22}$ Division of High Energy Physics, Department of Physics, University of Helsinki and Helsinki Institute of Physics, \\ FIN-00014, Helsinki, Finland \\ ${ }^{23}$ University of Illinois, Urbana, Illinois 61801, USA
}




\author{
${ }^{24}$ The Johns Hopkins University, Baltimore, Maryland 21218, USA \\ ${ }^{25}$ Institut für Experimentelle Kernphysik, Universität Karlsruhe, 76128 Karlsruhe, Germany \\ ${ }^{26}$ High Energy Accelerator Research Organization (KEK), Tsukuba, Ibaraki 305, Japan \\ ${ }^{27}$ Center for High Energy Physics: Kyungpook National University, Taegu 702-701, Korea; \\ Seoul National University, Seoul 151-742, Korea; \\ and SungKyunKwan University, Suwon 440-746, Korea \\ ${ }^{28}$ Ernest Orlando Lawrence Berkeley National Laboratory, Berkeley, California 94720, USA \\ ${ }^{29}$ University of Liverpool, Liverpool L69 7ZE, United Kingdom \\ ${ }^{30}$ University College London, London WC1E 6BT, United Kingdom \\ ${ }^{31}$ Centro de Investigaciones Energeticas Medioambientales y Tecnologicas, E-28040 Madrid, Spain \\ ${ }^{32}$ Massachusetts Institute of Technology, Cambridge, Massachusetts 02139, USA \\ ${ }^{33}$ Institute of Particle Physics: McGill University, Montréal, Canada H3A 2T8; \\ and University of Toronto, Toronto, Canada M5S $1 A 7$ \\ ${ }^{34}$ University of Michigan, Ann Arbor, Michigan 48109, USA \\ ${ }^{35}$ Michigan State University, East Lansing, Michigan 48824, USA \\ ${ }^{36}$ Institution for Theoretical and Experimental Physics, ITEP, Moscow 117259, Russia \\ ${ }^{37}$ University of New Mexico, Albuquerque, New Mexico 87131, USA \\ ${ }^{38}$ Northwestern University, Evanston, Illinois 60208, USA \\ ${ }^{39}$ The Ohio State University, Columbus, Ohio 43210, USA \\ ${ }^{40}$ Okayama University, Okayama 700-8530, Japan \\ ${ }^{41}$ Osaka City University, Osaka 588, Japan \\ ${ }^{42}$ University of Oxford, Oxford OXI 3RH, United Kingdom \\ ${ }^{43}$ University of Padova, Istituto Nazionale di Fisica Nucleare, Sezione di Padova-Trento, I-35131 Padova, Italy \\ ${ }^{44}$ LPNHE, Universite Pierre et Marie Curie/IN2P3-CNRS, UMR7585, Paris, F-75252 France \\ ${ }^{45}$ University of Pennsylvania, Philadelphia, Pennsylvania 19104, USA \\ ${ }^{46}$ Istituto Nazionale di Fisica Nucleare Pisa, Universities of Pisa, Siena \\ and Scuola Normale Superiore, I-56127 Pisa, Italy \\ ${ }^{47}$ University of Pittsburgh, Pittsburgh, Pennsylvania 15260, USA \\ ${ }^{48}$ Purdue University, West Lafayette, Indiana 47907, USA \\ ${ }^{49}$ University of Rochester, Rochester, New York 14627, USA \\ ${ }^{50}$ The Rockefeller University, New York, New York 10021, USA \\ ${ }^{51}$ Istituto Nazionale di Fisica Nucleare, Sezione di Roma 1, University of Rome "La Sapienza," I-00185 Roma, Italy \\ ${ }^{52}$ Rutgers University, Piscataway, New Jersey 08855, USA \\ ${ }^{53}$ Texas A\&M University, College Station, Texas 77843, USA \\ ${ }^{54}$ Istituto Nazionale di Fisica Nucleare, University of Triestel Udine, Italy \\ ${ }^{55}$ University of Tsukuba, Tsukuba, Ibaraki 305, Japan \\ ${ }^{56}$ Tufts University, Medford, Massachusetts 02155, USA \\ ${ }^{57}$ Waseda University, Tokyo 169, Japan \\ ${ }^{58}$ Wayne State University, Detroit, Michigan 48201, USA \\ ${ }^{59}$ University of Wisconsin, Madison, Wisconsin 53706, USA \\ ${ }^{60}$ Yale University, New Haven, Connecticut 06520, USA \\ (Received 28 December 2005; published 19 March 2007)
}

We present the first observation of the baryon decay $\Lambda_{b}^{0} \rightarrow \Lambda_{c}^{+} \pi^{-}$followed by $\Lambda_{c}^{+} \rightarrow p K^{-} \pi^{+}$in $106 \mathrm{pb}^{-1} p \bar{p}$ collisions at $\sqrt{s}=1.96 \mathrm{TeV}$ in the CDF experiment. In order to reduce systematic error, the measured rate for $\Lambda_{b}^{0}$ decay is normalized to the kinematically similar meson decay $\bar{B}^{0} \rightarrow D^{+} \pi^{-}$ followed by $D^{+} \rightarrow \pi^{+} K^{-} \pi^{+}$. We report the ratio of production cross sections $(\sigma)$ times the ratio of branching fractions $(\mathcal{B})$ for the momentum region integrated above $p_{T}>6 \mathrm{GeV} / c$ and pseudorapidity range $\quad|\eta|<1.3: \quad \sigma\left(p \bar{p} \rightarrow \Lambda_{b}^{0} X\right) / \sigma\left(p \bar{p} \rightarrow \bar{B}^{0} X\right) \times \mathcal{B}\left(\Lambda_{b}^{0} \rightarrow \Lambda_{c}^{+} \pi^{-}\right) / \mathcal{B}\left(\bar{B}^{0} \rightarrow D^{+} \pi^{-}\right)=0.82 \pm$ 0.08 (stat) \pm 0.11 (syst) $\pm 0.22\left[\mathcal{B}\left(\Lambda_{c}^{+} \rightarrow p K^{-} \pi^{+}\right)\right]$

DOI: $10.1103 /$ PhysRevLett.98.122002

PACS numbers: 14.20.Mr, 13.30.Eg, 14.65.Fy

Weak decays of baryons containing $b$ quarks are a good laboratory for testing the Heavy Quark Effective Theory (HQET) [1]. The $\Lambda_{b}^{0}$ baryon is the ground state of the $u d b$ quark system, and, in the heavy quark limit, the light degrees of freedom are in the state of zero total angular momentum [2]. Fully hadronic $b \rightarrow c \bar{u} d$ transitions are more complicated in baryons than in mesons because there are diagrams which are not present in the decays of the latter. Various extensions of HQET have been used to evaluate the $\Lambda_{b}^{0} \rightarrow \Lambda_{c}^{+} \pi^{-}$decay rate [3], but the predictions vary over a large range. However, in Soft Collinear Effective Theory (SCET) [4], all tree-level amplitudes 
can be properly evaluated, resulting in an explicit prediction for the ratio of branching fractions $\mathcal{B}\left(\Lambda_{b}^{0} \rightarrow\right.$ $\left.\Lambda_{c}^{+} \pi^{-}\right) / \mathcal{B}\left(\bar{B}^{0} \rightarrow D^{+} \pi^{-}\right) \approx 1.7$ [5]. The decays of $\Lambda_{b}^{0}$ are also interesting because they may provide a means to determine Cabibbo-Kobayashi-Maskawa (CKM) matrix elements with different systematic uncertainties than the determinations from the decays of $B$ mesons [6].

This is the first reconstruction of a hadronic decay of a $b$ baryon at a hadron collider that does not use a $J / \psi$ in the final state. In addition, our sample has more than an order of magnitude more events than any previous sample of fully reconstructed $\Lambda_{b}^{0}$ decays, and, for the same luminosity, is about 5 times larger than a sample of $\Lambda_{b} \rightarrow J / \psi \Lambda$ decays. Since $b$ baryons are not produced at the $B$ factories operating at the $Y(4 S)$ resonance, studying them comprises a unique facet of the $B$ physics program at Collider Detector at Fermilab (CDF II) [7]. In particular, a large sample of fully reconstructed $\Lambda_{b}^{0}$ decays would allow CDF to study other properties of $b$ baryons, e.g., to measure the lifetime of $\Lambda_{b}^{0}$, and also to search for decays of heavier $b$ baryons such as $\Sigma_{b}^{ \pm} \rightarrow \Lambda_{b}^{0} \pi^{ \pm}$.

This Letter presents a measurement of a ratio of $\Lambda_{b}^{0}$ and $\bar{B}^{0}$ branching fractions multiplied by the ratio of production cross-sections,

$$
R \equiv \frac{\sigma\left(p \bar{p} \rightarrow \Lambda_{b}^{0} X\right)}{\sigma\left(p \bar{p} \rightarrow \bar{B}^{0} X\right)} \frac{\mathcal{B}\left(\Lambda_{b}^{0} \rightarrow \Lambda_{c}^{+} \pi^{-}\right)}{\mathcal{B}\left(\bar{B}^{0} \rightarrow D^{+} \pi^{-}\right)},
$$

where the $\sigma$ quantities are the cross-sections for $\Lambda_{b}^{0}$ and $\bar{B}^{0}$ production in the pseudorapidity range $|\eta|<1.3$ with momentum in the transverse plane, $p_{T}$, above $6 \mathrm{GeV} / c$ [8]. This ratio compares the branching fractions of the topologically similar, fully reconstructed decays $\Lambda_{b}^{0} \rightarrow$ $\Lambda_{c}^{+} \pi^{-}$and $\bar{B}^{0} \rightarrow D^{+} \pi^{-}$, where the charmed hadrons decay via similar three-body channels $\Lambda_{c}^{+} \rightarrow p K^{-} \pi^{+}$ and $D^{+} \rightarrow \pi^{+} K^{-} \pi^{+}$[9]. The quantity $R$ is obtained from

$$
R=\frac{N_{\Lambda_{b}^{0}}}{N_{\bar{B}^{0}}} \frac{\mathcal{B}\left(D^{+} \rightarrow \pi^{+} K^{-} \pi^{+}\right)}{\mathcal{B}\left(\Lambda_{c}^{+} \rightarrow p K^{-} \pi^{+}\right)} \frac{\epsilon\left(\bar{B}^{0}\right)}{\epsilon\left(\Lambda_{b}^{0}\right)},
$$

where the first factor is the ratio of observed signal yields, the second factor is the ratio of the (external) daughter branching fractions [10], and the third factor is the ratio of reconstruction efficiencies calculated from the Monte Carlo simulations. To obtain $R$, the ratio of the reconstruction efficiencies of two topologically and kinematically similar decay modes is evaluated, reducing the systematic errors on the measured quantity.

The upgraded CDF II detector is well-suited for the detailed study of weak decays of heavy baryons. In particular, the advent of the Silicon Vertex Trigger (SVT) [11], which uses precise position measurements to select events containing weakly decaying heavy hadrons, allows CDF II to collect many hadronic decay modes of heavy baryons for the first time. This measurement is performed using a $106 \mathrm{pb}^{-1}$ sample of $p \bar{p}$ collisions collected by CDF II between February 2002 and June 2003. This data sample corresponds to $\sim 10^{10} b$ hadron decays produced in the central detector region. A full description of the CDF II detector can be found elsewhere [7]. The detector components pertinent to this analysis are the silicon microstrip vertex detector (SVX II) [12], the drift-chamber central tracker (COT) [13] and a three-tiered trigger system (Levels 1, 2, and 3). The five double-sided layers of the SVX II used in this analysis provide up to 10 position measurements. Of these, up to five are in the $r-\phi$ [8] plane (each precise to about $15 \mu \mathrm{m}$ ), three are longitudinal, and two are small-angle-stereo. The $\phi$ strips are parallel to the $z$-axis, longitudinal strips are inclined at $90^{\circ}$, and the small-angle-stereo strips are inclined at $1.2^{\circ}$. The SVX II spans the radii between 2.5 and $10.6 \mathrm{~cm}$ and covers the pseudorapidity range $|\eta|<2$. The COT has 96 measurement layers between the radii of 40 and $137 \mathrm{~cm}$. These are organized into alternating axial and small-angle-stereo $\left(2^{\circ}\right)$ superlayers. The COT has a smaller pseudorapidity coverage $(|\eta|<1.3)$ than the SVX II. Both tracking detectors are immersed in a $1.41 \mathrm{~T}$ magnetic field parallel to the $z$ axis.

This analysis, in particular, relies on the SVT, which operates as a part of the Level 2 trigger system. The trigger makes it possible to select events at a rate of $\sim 100 \mathrm{~Hz}$ from the $\sim 1 \mathrm{MHz}$ interaction rate. The components of the three level trigger system pertinent to this measurement are the Extremely Fast Tracker (XFT) at Level 1 and the SVT at Level 2. The XFT uses four axial superlayers of the COT to find tracks with $p_{T}>1.5 \mathrm{GeV} / c$. The SVT combines the XFT measurement with $r-\phi$ hits from the SVX II detector. The track finding is performed using a large lookup table of hit patterns. The found track candidates are fitted for curvature, angle projected onto the transverse plane and impact-parameter [14]. The impact-parameter measurement allows the selection of long-lived particles in the trigger decision.

The signal $\left(\Lambda_{b}^{0} \rightarrow \Lambda_{c}^{+} \pi^{-}\right)$and normalization $\left(\bar{B}^{0} \rightarrow\right.$ $D^{+} \pi^{-}$) events are collected using the same trigger. At Level 1, two tracks must satisfy $p_{T}>2.0 \mathrm{GeV} / c$, a scalar sum of transverse momenta $p_{T 1}+p_{T 2}>5.5 \mathrm{GeV} / c$, and an angular separation projected onto the transverse plane of $\Delta \phi<135^{\circ}$. At Level 2, the transverse momentum cuts are repeated, and it is required that each track has impact parameter $d_{0}>120 \mu \mathrm{m}$, with an angular separation between the tracks projected onto the transverse plane of $\left(2^{\circ}<\Delta \phi<90^{\circ}\right)$. Finally, the distance evaluated in the transverse plane from the primary vertex to the two-track intersection point must be greater than $200 \mu \mathrm{m}$.

Additional criteria are imposed on the triggered sample in order to reject as many background events as possible while keeping most of the signal. To reconstruct $\Lambda_{b}^{0} \rightarrow$ $\Lambda_{c}^{+} \pi^{-}$, minimum acceptable COT and SVX hit requirements are imposed, and all combinations of four tracks that pass such requirements are considered. Particle identification at $\mathrm{CDF}$ is possible only on a statistical basis and is not 
used in this analysis. The $p_{T}$ of the proton candidate from the $\Lambda_{c}^{+}$and the $\pi^{-}$candidate from the $\Lambda_{b}^{0}$ must be greater than $2.0 \mathrm{GeV} / c$, which strongly favors these particles to be the two which caused the event to pass the trigger. The $p_{T}$ of the proton candidate must be larger than the $p_{T}$ of the $\pi^{+}$candidate from the $\Lambda_{c}^{+}$. The $p_{T}$ of the $\Lambda_{b}^{0}$ and $\Lambda_{c}^{+}$ candidates must be greater than $7.5 \mathrm{GeV} / c$ and $4.5 \mathrm{GeV} / c$, respectively. In Eq. (1), $\sigma_{\Lambda_{b}^{0}}$ and $\sigma_{\bar{B}^{0}}$ are defined for $p_{T}>6 \mathrm{GeV} / c$. The events in the data sample must satisfy $p_{T}>7.5 \mathrm{GeV} / c$, and the difference is accounted for by using the Monte Carlo simulation based on the $p_{T}$ distributions of both $\Lambda_{b}^{0}$ and $\bar{B}^{0}$ measured in data [15].

Each of the unstable particles $\left(\Lambda_{b}^{0}, \bar{B}^{0}, \Lambda_{c}^{+}\right.$, and $\left.D^{+}\right)$is reconstructed by considering all valid combinations of tracks and requiring them to satisfy the decay hypothesis. The charmed hadrons $\Lambda_{c}^{+}$and $D^{+}$are reconstructed first: each triplet of tracks that satisfies the selection criteria (detailed below) is constrained to pass through the same point, called the decay vertex. The decay vertex is determined by varying the track parameters of the stable daughters within their uncertainties to minimize the $\chi^{2}$. The $\Lambda_{c}^{+}\left(D^{+}\right)$candidate is then combined with a fourth track to form a $\Lambda_{b}^{0}\left(\bar{B}^{0}\right)$ candidate. The full topology of the decay is then imposed in another kinematic fit, resulting in a simultaneous measurement of the $\Lambda_{b}^{0}\left(\bar{B}^{0}\right)$ and $\Lambda_{c}^{+}\left(D^{+}\right)$ vertices.

Using these measurements, the reconstructed invariant mass of the $\Lambda_{c}^{+}$must be between $2.269 \mathrm{GeV} / c^{2}$ and $2.301 \mathrm{GeV} / c^{2}$. Other selection criteria rely on $L_{x y}$, the projection onto the $x-y$ (transverse) plane of the decay length measured from the production vertex to the decay point; the production vertex is estimated by the position of the beam line averaged over each run calculated for the $z$ coordinate of the secondary vertex. A product of the proper decay time and the speed of light, $c t$, is also used. It is derived from $L_{x y}: c t=\left(\vec{L}_{x y} \cdot \hat{p}_{T}\right)\left(m c / p_{T}\right)$, where $\vec{L}_{x y}$ is the decay vector of $\Lambda_{b}^{0}\left(\bar{B}^{0}\right)$ projected onto the $x$-y plane, $p_{T}$ is the transverse momentum, $\hat{p}_{T}$ is the unit vector in the direction of the transverse momentum, and $m$ is the world average mass of the $\Lambda_{b}^{0}\left(\bar{B}^{0}\right)$. In order to suppress the combinatorial background from the interaction point, we impose $c t\left(\Lambda_{b}^{0}\right)>225 \mu \mathrm{m}$ (compared to the $b$ baryon mean decay length of $368 \pm 24 \mu \mathrm{m}$ [10]). Calculated relative to the $\Lambda_{b}^{0}$ decay point, $\operatorname{ct}\left(\Lambda_{c}^{+}\right)$must be $\operatorname{ct}\left(\Lambda_{c}^{+}\right)>-65 \mu \mathrm{m}$. For a true $\Lambda_{c}^{+}$, a small negative $c t$ may arise due to resolution effects; on the other hand, the $\operatorname{ct}\left(\Lambda_{c}^{+}\right)$of combinatorial background candidates may have large negative values. The distance of closest approach in the transverse plane of the trajectory of the $\Lambda_{b}^{0}$ candidate to the primary vertex must be less than $85 \mu \mathrm{m}$.

The normalization mode $\left(\bar{B}^{0} \rightarrow D^{+} \pi^{-}\right)$is reconstructed using selection criteria identical to those of the signal mode, except for a different invariant mass requirement for the $D^{+}$candidate and no analogy to the $p_{T}(p)>$

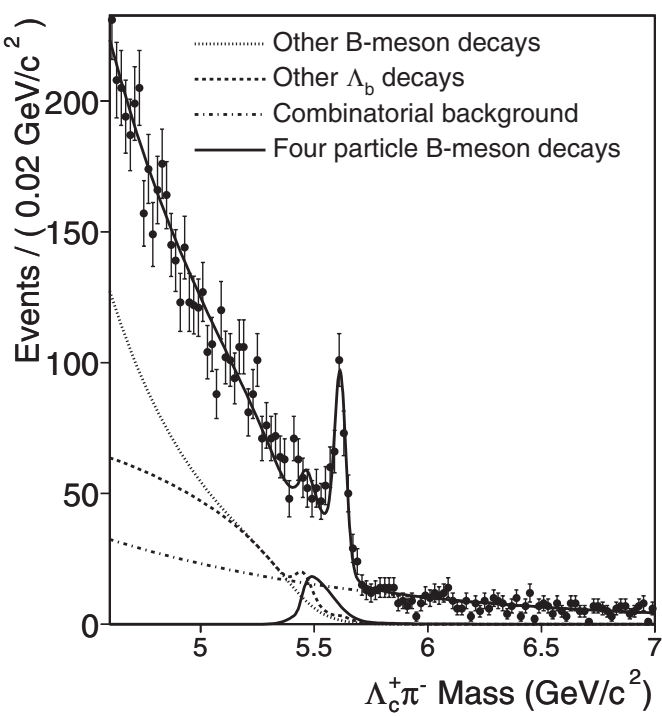

FIG. 1. $\quad \Lambda_{b}^{0} \rightarrow \Lambda_{c}^{+} \pi^{-}$yield with binned likelihood mass fit. The background shapes are defined in the text.

$p_{T}\left(\pi^{+}\right)$cut. The $D^{+}$candidate invariant mass must be between $1.848 \mathrm{GeV} / c^{2}$ and $1.888 \mathrm{GeV} / c^{2}$. The distributions of the invariant mass of $\Lambda_{b}^{0} \rightarrow \Lambda_{c}^{+} \pi^{-}$and $\bar{B}^{0} \rightarrow$ $D^{+} \pi^{-}$candidates are shown in Figs. 1 and 2, respectively.

Figure 1 shows the binned likelihood fit to the invariant mass distribution of $\Lambda_{b}^{0}$ candidates. The large Gaussian peak at $5.6 \mathrm{GeV} / c^{2}$ is the $\Lambda_{b}^{0} \rightarrow \Lambda_{c}^{+} \pi^{-}$signal. The dashdotted curve corresponds to the exponential combinatorial background. This component is constrained by the data in the invariant mass region above the $\Lambda_{b}^{0}$ mass. The small asymmetric peak at $5.5 \mathrm{GeV} / c^{2}$ (solid line) corresponds to contributions from fully reconstructed $B$-meson decays resulting in a final state with four tracks, where at least

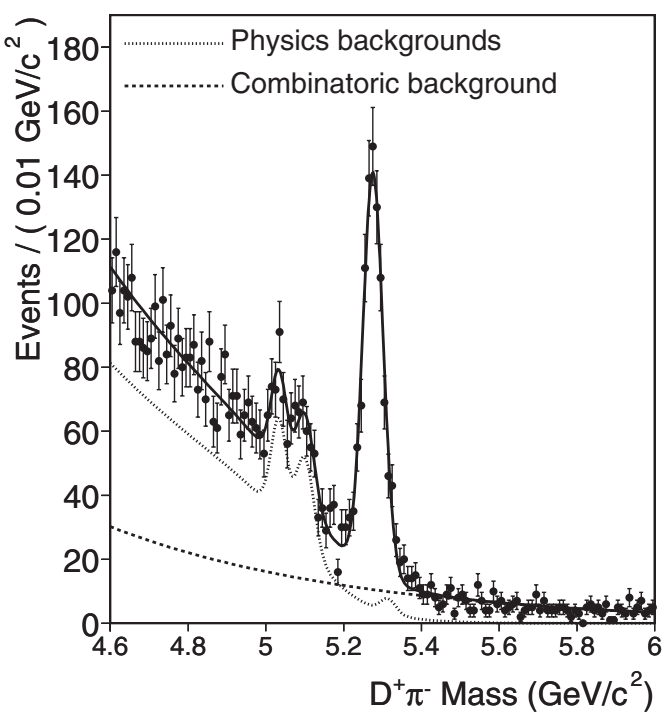

FIG. 2. $\quad \bar{B}^{0} \rightarrow D^{+} \pi^{-}$yield with binned likelihood fit. The background shapes are defined in the text. 
one track is misidentified. This shape is obtained using a full detector simulation of these modes. It is consistent with the shape of $\bar{B}^{0} \rightarrow D^{+} \pi^{-}$candidates found in the $\Lambda_{b}^{0}$ sample. The dotted and dashed curves correspond to all the other $B$-meson and $\Lambda_{b}^{0}$ backgrounds, respectively. These shapes are determined from a large parametric Monte Carlo sample which includes all known decays of $B^{+}, B^{0}$, and $B_{s}$ and $\Lambda_{b}^{0}$ hadrons. Finally, there is a very small Gaussian distribution (not shown) from the Cabibbosuppressed mode $\Lambda_{b}^{0} \rightarrow \Lambda_{c}^{+} K^{-}$(fixed to an expected $8 \%$ of the signal yield [16]). The total distribution is $F_{\text {tot }}=$ $G_{\text {signal }}+E_{\text {comb }}+F_{\text {four-track }}+F_{\text {other- } B}+F_{\text {other- } \Lambda_{b}^{0}}+G_{\Lambda_{c}^{+} K^{-}}$, where $G$ indicates a Gaussian distribution, $E$ indicates an exponential, and $F$ indicates a more complicated functional form.

In the fit, the width of $\Lambda_{b}^{0}$ signal is fixed to $26.4 \mathrm{MeV} / c^{2}$, obtained by scaling the $\bar{B}^{0}$ width in data by the ratio of widths from the Monte Carlo simulation. The relative contribution of each background type in the fit is guided by two constraints: the first describes the normalization of $F_{\text {four-track }}$ relative to $F_{\text {other- } B}$ (i.e., $N_{\text {four-track }} / N_{\text {other- } B}$ ), where $N$ is number of events; the second describes the normalization of $F_{\text {other- } \Lambda_{b}^{0}}$ relative to $\left(F_{\text {other- } B}+F_{\text {four-track }}\right)$ (i.e., $\left.N_{\text {other } \Lambda_{b}^{0}} /\left(N_{\text {four-track }}+N_{\text {other- } B}\right)\right)$. The value of each constraint is inferred from the relative abundance of the background types in the large parametric Monte Carlo sample. The value of the $\left(N_{\text {four-track }} / N_{\text {other- } B}\right)$ constraint is checked by reconstructing the $\bar{B}^{0} \rightarrow D^{+} \pi^{-}$mode among $\Lambda_{c}^{+} \pi^{-}$candidates from the region of the invariant mass corresponding to the $\Lambda_{b}^{0}$ signal. The total $\chi^{2}$ of this fit is 80.6 for 88 degrees of freedom, corresponding to the fit probability of $70 \%$.

Figure 2 shows the mass fit for the $D^{+} \pi^{-}$candidates. The large Gaussian peak at $5.27 \mathrm{GeV} / c^{2}$ is the $\bar{B}^{0}$ signal. The dashed curve corresponds to the exponential combinatorial background. The dotted curve corresponds to all physics backgrounds, both from $B^{0}$ decays (including $\bar{B}^{0} \rightarrow D^{+} K^{-}$) as well as decays of other $b$ hadrons. The total $\chi^{2}$ of this fit is 70.9 for 94 degrees of freedom, corresponding to the fit probability of $96 \%$.

The quantity $R$ defined in Eq. (1) is calculated from the signal yields according to (2). The signal yields are $N_{\Lambda_{b}^{0}}=$ $214 \pm 19$ and $N_{\bar{B}^{0}}=790 \pm 32$, respectively. Each reconstruction efficiency is defined for the $\Lambda_{b}^{0}\left(\bar{B}^{0}\right)$ with $p_{T}>$ $6 \mathrm{GeV} / c$ and $|\eta|<1.3$. The exact configuration of the CDF II detector varied over the course of collecting the data used in the analysis, with the average efficiency of $\sim 0.5 \%$. However, the ratio of reconstruction efficiencies is stable within statistical errors across the different periods of running, with the average value $\epsilon\left(\bar{B}^{0}\right) / \epsilon\left(\Lambda_{b}^{0}\right)=1.65 \pm$ 0.03 (stat). From Eq. (2) we thus obtain $R=0.82 \pm$ 0.08 (stat).

The systematic uncertainty on the measurement of $R$ is dominated by the error on $\mathcal{B}\left(\Lambda_{c}^{+} \rightarrow p K^{-} \pi^{+}\right)$, yielding a relative error of $27 \%$ [10]. Since this uncertainty is independent of our measurement, it is quoted separately. The bulk of the remaining systematic uncertainty (12.1\%) comes from $\epsilon\left(\bar{B}^{0}\right) / \epsilon\left(\Lambda_{b}^{0}\right)$. It arises from the imperfect knowledge of the $\Lambda_{b}^{0}$ lifetime $\left({ }_{-4}^{+5} \%\right)$, the production $p_{T}$ spectra of both $\Lambda_{b}^{0}(7.6 \%)$ and $\bar{B}^{0}(4 \%)$, the $\Lambda_{b}^{0}$ polarization (7\%), and the $\Lambda_{c}^{+}$resonant substructure (1\%). The uncertainty due to the finite size of the Monte Carlo samples is $1.9 \%$. The uncertainty due to the difference between the proton and $\pi^{+}$trigger efficiency is $0.6 \%$. The systematic uncertainty on $N_{\Lambda_{b}^{0}} / N_{\bar{B}^{0}}(5.7 \%)$ is largely due to a lack of detailed knowledge of a variety of branching fractions contributing to background shapes that are obtained from the Monte Carlo simulation and fixed in the fit. To evaluate the uncertainty due to these shapes, the branching fractions of the largest decay modes contributing to each of the shapes were varied simultaneously in the simulation, and the shapes were reevaluated. This procedure yields uncertainties of $4.3 \%$ and $0.9 \%$ for the $\Lambda_{b}^{0}$ and the $\bar{B}^{0}$ mass fits, respectively. Uncertainties on the mass resolutions of both $\Lambda_{b}^{0}$ and $\bar{B}^{0}$, which are also fixed in the mass fits, contribute $2.8 \%$ and $1.8 \%$, respectively. Finally, the contribution of the $\Lambda_{b}^{0} \rightarrow \Lambda_{c}^{+} K^{-}$shape is varied by a factor of 2, contributing $1.6 \%$ to the systematic error. The total systematic error excluding the uncertainty on $\mathcal{B}\left(\Lambda_{c}^{+} \rightarrow p K^{-} \pi^{+}\right)$is $13.5 \%$.

A direct comparison with a theoretical prediction of $R_{\mathrm{BR}} \equiv \mathcal{B}\left(\Lambda_{b}^{0} \rightarrow \Lambda_{c}^{+} \pi^{-}\right) / \mathcal{B}\left(\bar{B}^{0} \rightarrow D^{+} \pi^{-}\right) \approx 1.7$ [5] can be performed if one assumes that $\sigma\left(\Lambda_{b}\right)$ and $\sigma\left(B^{0}\right)$ have the same dependence on $p_{T}$, and then use $f_{\text {baryon }} / f_{d}$ from high- $p_{T}$ measurements. From Ref. [10], we obtain $f_{\text {baryon }} / f_{d}=0.25 \pm 0.04, \quad$ yielding $\quad R_{\mathrm{BR}} \approx 3.3 \pm 1.2$, which compares well with [5].

In summary, we have observed the decay $\Lambda_{b}^{0} \rightarrow \Lambda_{c}^{+} \pi^{-}$ for the first time, and measured $R=0.82 \pm 0.08$ (stat) \pm 0.11 (syst) $\pm 0.22\left(\mathcal{B}_{\Lambda_{c}^{+}}\right)$. The overall error is dominated by the large uncertainty on $\mathcal{B}\left(\Lambda_{c}^{+} \rightarrow p K^{-} \pi^{+}\right)$. The $\Lambda_{b}^{0} \rightarrow$ $\Lambda_{c}^{+} \pi^{-}$sample is the largest $b$-baryon sample in existence, and, once augmented by new data, can be used for a variety of other $\Lambda_{b}$ measurements, including its lifetime and production properties.

We thank the Fermilab staff and the technical staffs of the participating institutions for their vital contributions. This work was supported by the U.S. Department of Energy and National Science Foundation; the Italian Istituto Nazionale di Fisica Nucleare; the Ministry of Education, Culture, Sports, Science and Technology of Japan; the Natural Sciences and Engineering Research Council of Canada; the National Science Council of the Republic of China; the Swiss National Science Foundation; the A.P. Sloan Foundation; the Bundesministerium für Bildung und Forschung, Germany; the Korean Science and Engineering Foundation and the Korean Research Foundation; the Particle Physics and Astronomy Research Council and the Royal Society, UK; 
the Russian Foundation for Basic Research; the Comisión Interministerial de Ciencia y Tecnología, Spain; in part by the European Community's Human Potential Programme under Contract No. HPRN-CT-2002-00292; and the Academy of Finland.

[1] For a review, see A. F. Falk, "Introduction to hadronic B physics," published in The BABAR Physics Book: Physics at an Asymmetric B Factory, No. SLAC-R-504; or A. V. Manohar and M.B. Wise, Heavy Quark Physics (Cambridge University Press, Cambridge, 2000).

[2] A. F. Falk and M. Neubert, Phys. Rev. D 47, 2982 (1993).

[3] X. H. Guo, Mod. Phys. Lett. A 13, 2265 (1998).

[4] C. W. Bauer, S. Fleming, and M. E. Luke, Phys. Rev. D 63, 014006 (2000); C. W. Bauer, S. Fleming, D. Pirjol, and I. W. Stewart, Phys. Rev. D 63, 114020 (2001); C. W. Bauer and I. W. Stewart, Phys. Lett. B 516, 134 (2001); C. W. Bauer, D. Pirjol, and I. W. Stewart, Phys. Rev. D 65 , 054022 (2002).

[5] A. K. Leibovich, Z. Ligeti, I. W. Stewart, and M. B. Wise, Phys. Lett. B 586, 337 (2004).

[6] I. Dunietz, Z. Phys. C 56, 129 (1992).

[7] D. Acosta et al., Phys. Rev. D 71, 032001 (2005)

[8] CDF II uses a cylindrical coordinate system with the $z$-axis along the nominal beam line. The transverse plane $(x, y)$ is perpendicular to the $z$-axis. Azimuthal angle, $\phi$, is measured from the $x$-axis. Polar angle, $\theta$, is measured from the $z$-axis. Pseudorapidity is defined as $\eta \equiv$ $\tanh ^{-1}(\cos \theta)$. Transverse momentum, $p_{T}$, is the component of the particle's momentum projected onto the transverse plane.

[9] Inclusion of the respective charge conjugate modes is assumed throughout this Letter. Specifically, $\bar{\Lambda}_{b}^{0} \rightarrow$ $\Lambda_{c}^{-} \pi^{+}, \quad B^{0} \rightarrow D^{-} \pi^{+}, \quad \Lambda_{c}^{-} \rightarrow \bar{p} K^{+} \pi^{-}, \quad$ and $\quad D^{-} \rightarrow$ $\pi^{-} K^{+} \pi^{-}$.

[10] S. Eidelman et al., Phys. Lett. B 592, 1 (2004).

[11] W. Ashmanskas et al., Nucl. Instrum. Methods Phys. Res., Sect. A 518, 532 (2004).

[12] A. Sill et al., Nucl. Instrum. Methods Phys. Res., Sect. A 447, 1 (2000).

[13] A. Affolder et al., Nucl. Instrum. Methods Phys. Res., Sect. A 526, 249 (2004).

[14] The curvature of a particle track is inversely proportional to the transverse momentum $\left(p_{T}\right)$. The impact-parameter of a particle track is defined as the distance of closest approach of the particle track to the primary vertex in the transverse plane.

[15] S. Yu, Ph.D. thesis, University of Pennsylvania, 2005. hepex/0504059.

[16] This choice is motivated by K. Abe et al., Phys. Rev. Lett. 87, 111801 (2001); however, we assign a large systematic error to it. 